\title{
Low Order Modelling of Lift Forces for Unsteady Pitching and Surging Wings.
}

\author{
H. Babinsky*, P. R. R. J. Stevens ${ }^{\dagger}$ \\ Department of Engineering, University of Cambridge, CB2 1PZ, United Kingdom. \\ A. R. Jones ${ }^{\ddagger}$ \\ Department of Aerospace Engineering, University of Maryland, College Park, MD 20742, United States of America. \\ L. P. Bernal ${ }^{\S}$ \\ University of Michigan, Ann Arbor, MI, 48109, United States of America. \\ M. V. Ol ${ }^{\mathbb{I}}$ \\ U.S. Air Force Research Laboratory, Wright-Patterson AFB, OH, 45433, United States of America.
}

\begin{abstract}
This article describes a simple low-order model for the lift produced by rapidly accelerating and pitching flat plate wings. The model is informed by experiments performed as part of NATO's AVT-202 technical team. The overall agreement of the model with the forces measured as part of this effort is reasonable, however, the main value of the model is to identify a number of contributions to the lift force and classifying these as either circulatory or non-circulatory. Thus the relative effects of viscosity and kinematic accelerations are identified which helps the understanding of unsteady low-Re flows.
\end{abstract}

\section{Introduction}

The work reported here was performed under the umbrella of NATO's RTO task group AVT-202 'Advances in Fundamental Unsteady Low Reynolds Number Flows'. An overview of the task group and results by various members of the group are presented in a number of companion papers at the same conference. One of the aims of the task group was to identify the contributions to unsteady lift due to circulatory and noncirculatory effect. To this aim, several basic canonical test cases were studied. Here, the results from a subset of these tests (see Figure 1) are used to develop a very simple analytical method to predict the lift generated by a flat plate wing in an unsteady flow. Two types of unsteady flow are considered, a flat plate accelerating from rest at a constant angle of incidence and a flat plate pitching in a steady free-stream. In both cases, the steady state angle of incidence is $45^{\circ}$. The flow is treated as 2-dimensional on the assumption that end effects are small for rapid unsteady motions of plates with aspect ratios of the order 4 or above.

By devising a low-order model and comparing its predictions to the experimentally observed force histories it is possible to identify the most significant contributions to lift and to separate circulatory from non-circulatory effects.

\footnotetext{
*Professor of Aerodynamics, Cambridge University Engineering Department Aerodynamics Lab, Associate Fellow AIAA.

${ }^{\dagger}$ Research Associate, Cambridge University Engineering Department Aerodynamics Lab, Member AIAA.

$\ddagger$ Assistant Professor, Department of Engineering, University of Maryland, College Park, Associate Fellow AIAA.

\$Professor, Department of Aerospace Engineering, University of Michigan, Senior Member AIAA.

IITechnical Advisor, Aero Configurations Branch, Aerospace Systems Directorate, Associate Fellow AIAA.
} 


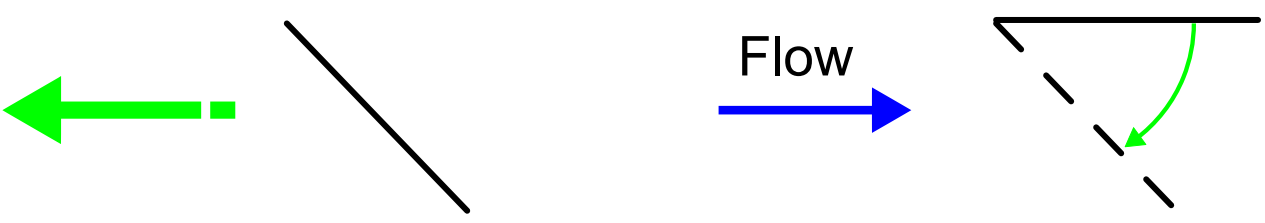

Figure 1: The two fundamental AVT-202 test cases: Acceleration from rest ('sliding wing') and pitching in a constant freestream ('pitching wing').

\section{A. Low-order modelling of unsteady lift}

At the start we accept without proof that the unsteady force exerted by an object onto the surrounding fluid (and thus the opposite reaction force experienced by the object) is equal to the rate of change of momentum in the surrounding fluid. To quantify this force it is necessary to integrate the momentum across the whole flowfield (stretching to infinity) and then calculate the time derivative ${ }^{a}$ :

$$
F=\frac{d}{d t} \iint_{-\infty}^{\infty} \rho \boldsymbol{u}(x, t) d A
$$

where $u$ is the unsteady velocity field, $x$ is the location vector, $t$ is time and $A$ is an area element $(d x d y)$. Thus, if it were possible to accurately determine the velocity field everywhere at several instances in time one could determine the force experienced by an object i.e. lift and drag. In reality however, there are considerable practical difficulties to this approach.

In any case, it is helpful to break the overall force into several contributions due to inertial and vortex effects as this can reveal interesting insights and can provide guidance for future designs.

\section{Inertial effects: Virtual mass}

Returning to Eq. 1 we can make some further observations for situations where the unsteady flow around an object is self-similar. By this we mean a flowfield where the streamline pattern is independent of time although the magnitude of local velocity may scale with a time-varying free-stream $U_{\infty}(t)$ :

$$
u(x, t)=u_{\infty}(t) k(x)
$$

Here $k(x)$ is some function relating the local velocity to the (variable) free stream. Note that $k$ is constant in time. Thus, the force experienced by the object can be expressed as follows:

$$
\boldsymbol{F}=\underbrace{\rho \iint_{-\infty}^{\infty} k(x) d A}_{I} \underbrace{\frac{d U_{\infty}(t)}{d t}}_{I I}
$$

The force is therefore the product of a time-invariant area integral of $\mathrm{k}$ (Term I) and the acceleration of the free-stream (Term II). Note that the first term (Term I) has the unit of mass and is constant throughout the motion. Thus the force is proportional to the acceleration.

A good way to illustrate this force contribution is to consider the inviscid flow around a flat plate at $90^{\circ}$ angle of incidence, as seen in Figure 2.

In steady flow there is no drag force. However, in unsteady flow the surface pressures on the windward side are increased, while the pressures at the rear are decreased, giving a net force. This force can be derived from unsteady potential flow results:

$$
F=\frac{\rho c^{2} \pi}{4} \dot{V}
$$

where $c$ is the chord of the plate and $V$ the plate-normal velocity of the fluid.

This force is exactly equivalent to the inertia of a circular body of fluid with diameter c undergoing the same motion as the plate. Therefore, this force is often referred to as an 'added' mass force (or 'virtual' mass

\footnotetext{
${ }^{\text {a }}$ The pressure is assumed uniform at the integration boundary $(\infty)$. Vector quantities are in bold.
} 


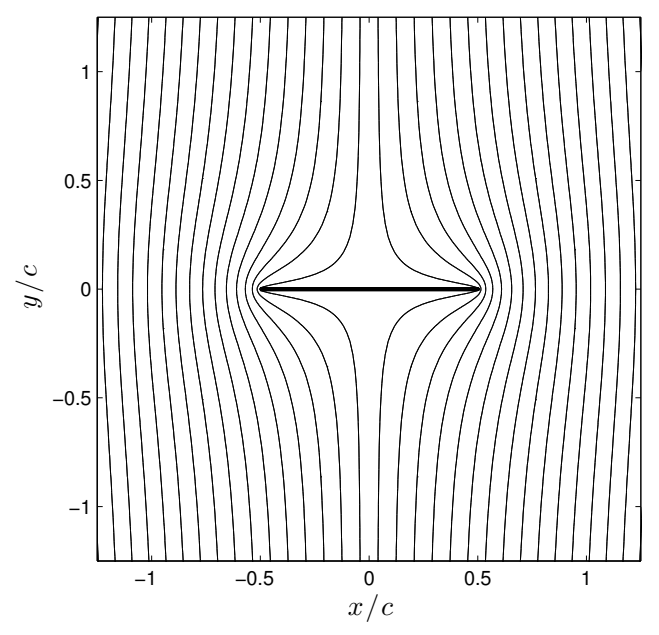

Figure 2: Potential flow around a flat plate at $90^{\circ}$ angle of incidence.

force). For a plate accelerating at an angle of incidence different from $90^{\circ}$, only the velocity component normal to the plate contributes to this force (by the principle of superposition).

There are more rigorous derivations of virtual mass which show that, even in a viscous (incompressible) flowfield, featuring separations and vortices, the inertial response to an acceleration is correctly captured by the above approach. ${ }^{1}$ This is because the inertial force is transmitted through pressure waves (moving at infinite speed in incompressible flow) which affect the whole flowfield in the same manner for potential or real viscous flows.

In the unsteady flowfields discussed below (which are not inviscid) we will therefore determine the virtual mass contribution by considering the plate-normal velocity at mid-chord. The force magnitude can then be calculated from the added mass force experienced by a flat plate at $90^{\circ}$ angle of incidence in unsteady potential flow, with the same plate-normal acceleration.

\section{Vortex lift (circulatory force)}

In a real flowfield viscosity can not be neglected. We thus postulate that the total unsteady force is the sum of the added mass force and a circulatory force contribution.

The simplest model for a circulatory force is to consider the relationship between forces and vortices. The flow shown in Figure 3 features a pair of equal, opposite-strength vortices. Such a flowfield has net momentum. Thus the generation of a pair of vortices in a quiescent fluid requires a force to introduce this momentum. According to $\mathrm{Lamb}^{2}$ the magnitude of this momentum is:

$$
J=\rho \Gamma d
$$

where $J$ is the impulse (momentum), $d$ the distance between the vortex centres and $\Gamma$ is the vortex circulation. Since force equals the rate of change of momentum, the chain rule dictates that:

$$
F=\rho(\Gamma \dot{d}+\dot{\Gamma} d)
$$

(where dot signifies time derivatives). The direction of this force is normal to the line connecting the two vortices.

This shows that unsteady vortex lift has two contributions, one determined by the strength of the vortices and the growth in distance between them (this term reduces to $L=\rho U \Gamma$ in steady flow) and another which is directly related to the growth rate of circulation.

We consider a flat plate wing in unsteady flow as being the source of such a vortex force through the generation of either a bound circulation or a leading edge vortex (LEV) and a corresponding starting or trailing edge vortex (TEV). For now the bound vortex and/or the LEV are effectively combined into a single vortex, here labelled LEV for simplicity. Setting $x$ as the streamwise co-ordinate, lift is the $y$-component 


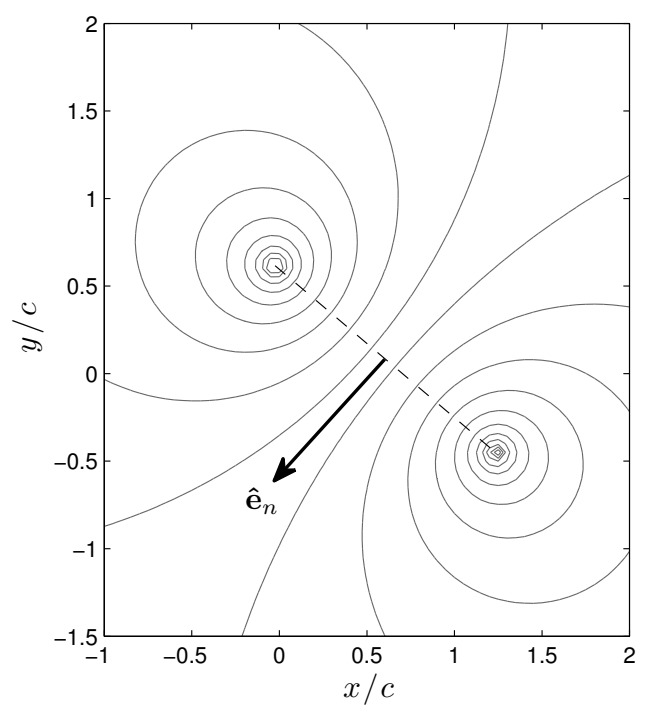

Figure 3: Potential flow streamlines around a stationary counter-rotating vortex pair. This flow has net momentum.

of this force. Knowledge of the strengths and positions of the two vortices (LEV and TEV) allows us to compute the circulatory lift force and express it in coefficient form (where $u_{i}$ are the $x$-components of vortex velocities, and $x_{i}$ are the $x$-components of vortex locations):

$$
c_{l, \text { circ }}=-\frac{2}{U_{\infty}^{2} c}\left[\left(u_{L E V}-u_{T E V}\right) \Gamma_{L E V}+\left(x_{L E V}-x_{T E V}\right) \dot{\Gamma}_{L E V}\right]
$$

Note that the lift coefficient in Eq. 7 is non-dimensionalised by a free stream velocity $U_{\infty}$. Apart from the choice of $U_{\infty}$ and the direction of the lift force this result is independent of the co-ordinate system.

In situations were there is both bound circulation and a LEV it is suggested that we 'lump together' both vortices by calculating the total circulation and a centroid location (comparable to a centre of gravity). Note that the position of the centroid can move relative to the plate (if the LEV moves away from the wing). The same principle is applied if multiple LEVs are shed from a wing and the totality of vorticity shed from the trailing edge is also assumed to be concentrated in just a single TEV. Thus, there are only two vortices in the flow and these must be of equal and opposite strength according to Kelvins theorem. In reality, as the LEV changes strength, a vortex sheet is shed from the trailing edge, which may not always merge with the TEV (and even if it does this will take time) and thus the above flow is a relatively crude approximation ${ }^{\mathrm{b}}$.

However, some of the above can be taken into account by replacing the distance $\left(x_{L E V}-x_{T E V}\right)$ in the second term of Eq. 7 with the chord length $c$. The reasoning for this is that whenever the LEV circulation changes, vorticity is shed from the trailing edge, thus approximately one chord length away from the LEV location. At high angles of incidence, this should be adjusted to $c \cdot \cos \alpha$, to reflect the horizontally projected distance.

Although rather basic, this model is helpful in understanding the physics of circulatory lift generated in an unsteady wing flow. For example, it illustrates clearly that a detached LEV can contribute to wing lift under two conditions: A) while it continues to strengthen, regardless of its position, and B) while it moves relative to the TEV (drifiting away from the wing at a slower speed than the TEV). Generally, after the LEV is 'shed' it stops growing in strength and moves away from the wing at an ever increasing rate (thus reducing its force contribution) until it ultimately drifts at free stream velocity at which point no further force is produced.

We now proceed to develop very simple models for the unsteady lift force produced by pitching and surging wings. In each case it is assumed that the total force is composed of an added mass contribution and a circulatory force. The added mass contribution is calculated from the potential flow around a flat

\footnotetext{
${ }^{\mathrm{b}}$ Assuming that the vortex sheet moves away with $U_{\infty}$, the first term of the above equation remains correct.
} 
plate normal to the flow and the circulatory contribution is derived from the two vortex model discussed above.

\section{Surging Wing}

\section{A. Non-circulatory force}

Following on from the above we express the plate-normal velocity as:

$$
V=U \sin \alpha
$$

Thus, from above, the added mass force (which is perpendicular to the plate) is:

$$
F=\frac{\rho c^{2} \pi}{4} \dot{U} \sin \alpha
$$

The component in lift direction

$$
L=F \cos \alpha=\frac{\rho c^{2} \pi}{4} \dot{U} \sin \alpha \cdot \cos \alpha
$$

can also be expressed as coefficient:

$$
C_{L, n o n-c i r c}=\frac{\pi c \dot{U}}{4 U^{2}} \sin 2 \alpha
$$

It is generally more useful to normalise the force coefficients in the surging case with the eventual free-stream velocity $U_{\infty}$.

\section{B. Circulatory force}

To determine the circulatory force contribution we make use of Pitt-Ford's observation that the bound circulation in surging flat plates tends to vanish early on in the motion. ${ }^{3}$ Thus we assume that all circulation in the flow is concentrated in two vortices: an LEV (that need not be attached to the plate) and a TEV.

To accurately calculate the circulatory force it is necessary to predict or measure the strength and location of the LEV as a function of time. For impulsively started thin airfoils at moderate angles of incidence, Wagner calculated the growth of bound circulation and lift as shown in Figure $4 .{ }^{4}$ It can be seen that the bound circulation grows from an initial value of zero to asymptotically reach the steady state value after many chord lengths of travel. The exact Wagner function is iterative but a close fit explicit formula is:

$$
\frac{\Gamma(t)}{\Gamma_{\infty}}=0.914-0.3151 \exp \left(\frac{s / c}{0.1824}\right)-0.5986 \exp \left(\frac{s / c}{2.0282}\right)
$$

In contrast, the lift starts at a finite value (equivalent to half of the steady state result). The reason for this apparent discrepancy is explained by Eq. 6: While bound circulation is growing the total lift is composed not only of the traditional $\rho U \Gamma$ but also of an unsteady contribution proportional to the growth rate of bound circulation, $\dot{\Gamma}$. In Wagner's calculations this has a finite non-zero value at $t=0$, causing the lift force observed at the start of the motion.

Although Wagner's calculations were derived for the bound circulation on an airfoil at small angles of incidence, his prediction of the circulation growth has been found to also describe the growth of a LEV in surging flat plate wings at high alpha, ${ }^{3}$ as seen in Figure 5. Here, the measured LEV circulation fits reasonably well with a Wagner curve (Eq. 12), except during the early part of the motion. One explanation for the discrepancy is that the experimental data was obtained on a wing that accelerated for a given time (equivalent to one chord length of travel) whereas Wagners function was determined for a theoretical instantaneous start. 


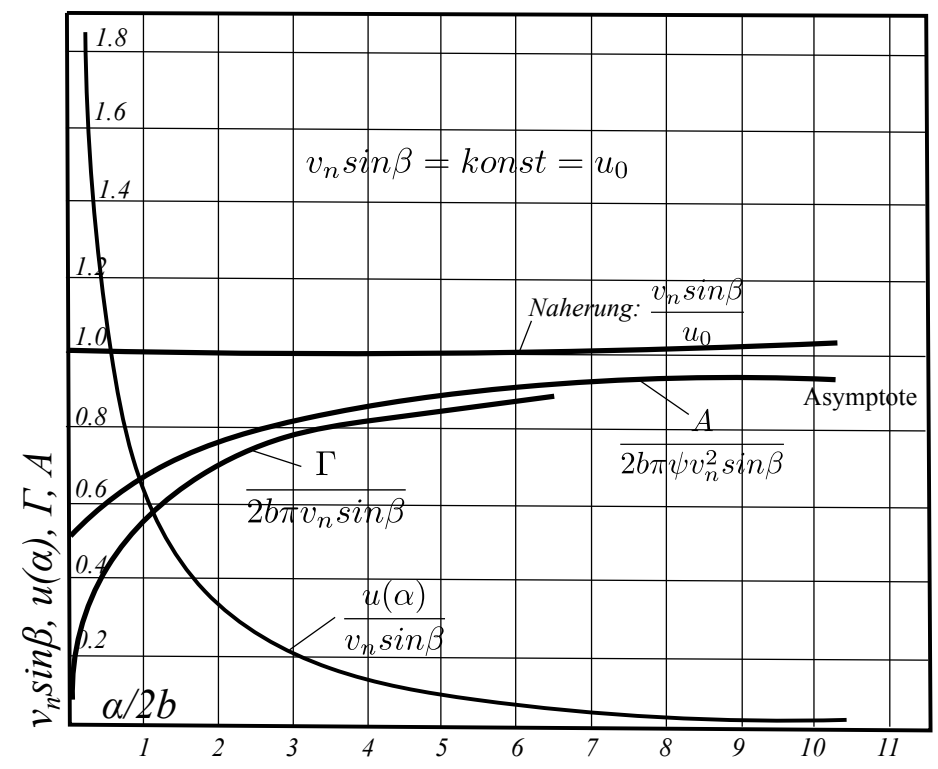

Figure 4: Wagner's force prediction (starting at 0.5) and Wagner's bound circulation (starting from 0). Reproduced from Wagner's original figure. ${ }^{4}$

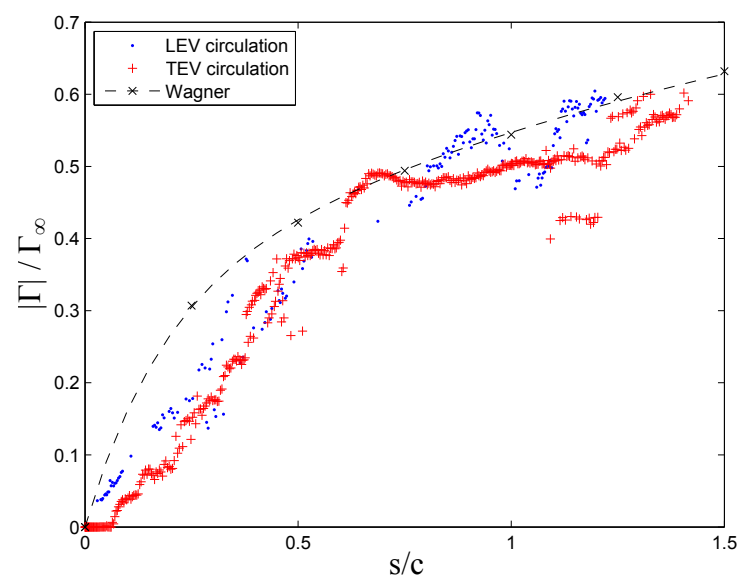

(a) $\alpha=15^{\circ}$.

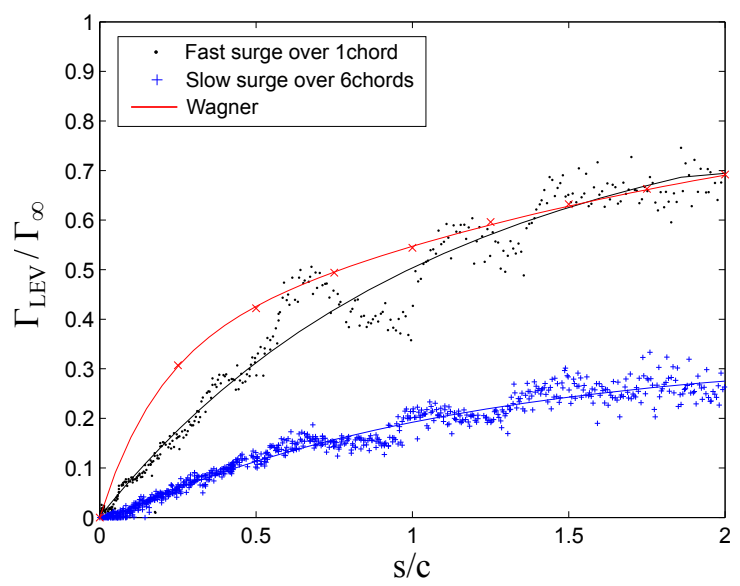

(b) $\alpha=45^{\circ}$.

Figure 5: LEV circulation for surging flat plates at $15^{\circ}$ and $45^{\circ}$ angle of incidence. ${ }^{3}$

Thus, the agreement can be improved during the acceleration phase by scaling the Wagner function according to the actual plate velocity, i.e. reducing the predicted circulation by $U(t) / U_{\infty}$ during the acceleration phase to give:

$$
\frac{\Gamma(t)}{\Gamma_{\infty}}=\frac{U(t)}{U_{\infty}}\left[0.914-0.3151 \exp \left(\frac{s / c}{0.1824}\right)-0.5986 \exp \left(\frac{s / c}{2.0282}\right)\right]
$$

In order to apply this 'modified Wagner' prediction to a LEV it is necessary to define the ultimate steadystate LEV circulation, $\Gamma_{\infty}$. Here, it is suggested to simply use a value equivalent to $c_{L}=2 \pi \alpha$ because it has been found to match the experimental data reasonably well. Note that this assumption does not imply that the plate actually achieves the equivalent value of lift, because the LEV does not remain attached to the 
plate. Instead the LEV moves downstream which reduces the relative velocity between the LEV and the TEV and thus limits the lift according to Eq. 7.

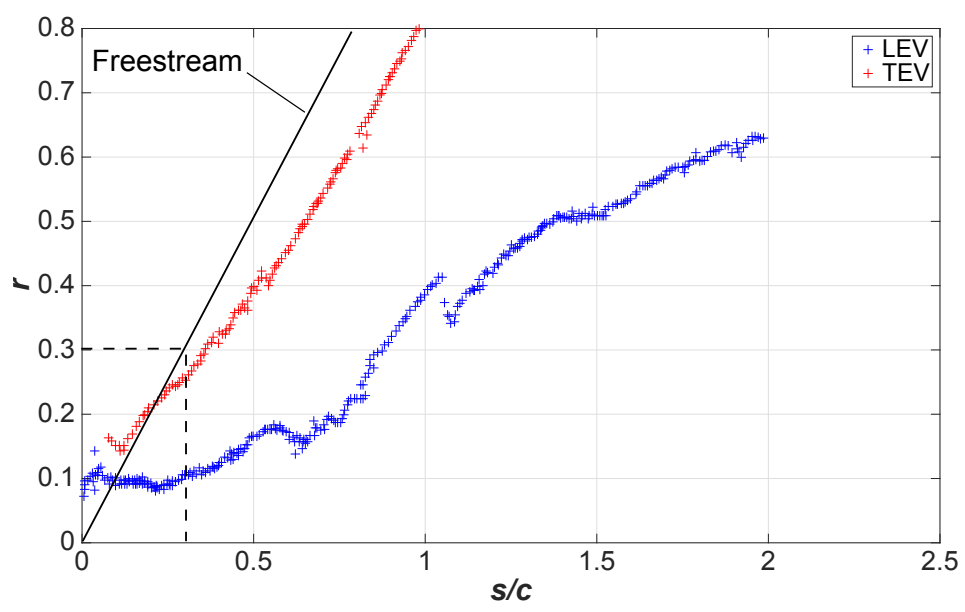

Figure 6: LEV and TEV distance from wing leading edge for fast surge case.

We also need an estimate of the LEV motion relative to the plate (or the TEV). Figure 6 shows some sample data from the AVT202 fast pitch and surge cases where the distance between the LEV/TEV cores and the wing leading edge $r$ are plotted against reduced time (equivalent to distance travelled by the wing). The free-stream velocity is marked by a solid line. It can be seen that the LEV trajectory converges onto a 'drift' velocity, which is roughly half of the free-stream. In contrast, the TEV exhibits different behaviour for surging and pitching wings but eventually both cases also converge to a common TEV trajectory moving away from the wing at free-stream speed. Thus, we can roughly estimate the relative drift velocity between the LEV and the TEV to be half of the free stream velocity. A long-term adjustment to this might also be considered because this can not hold indefinitely as the relative velocity has to vanish once the LEV has completely detached from the wing.

To summarise, we propose to estimate the unsteady force in the surge case as follows:

1. Add three contributions from virtual mass, vortex growth $\dot{\Gamma}_{L E V}$ and relative LEV/TEV motion.

2. Determine the virtual mass from Eq. 11. This force occurs during the accelerating portion of the cycle.

3. Estimate the two circulatory contributions by assuming that $\Gamma_{L E V}$ grows according to the speedadjusted Wagner function (Eq. 13) with a steady state value equivalent to $2 \pi \alpha$.

4. Estimate the LEV trajectory by assuming that the distance between the LEV and TEV grows at $50 \%$ of $U_{\infty}$.

5. Estimate the distance between two vortices used in the $\dot{\Gamma}_{L E V}$ term to equate to $c \cdot \cos \alpha$ (to a first approximation).

6. In each case, non-dimensionalise the result by the asymptotic $U_{\infty}$.

At large angles of incidence it is necessary to carefully consider the directions of the various forces and include a trigonometric term. The vortex lift acts in a direction normal to the line connecting LEV and $\mathrm{TEV}$, while the non-circulatory force acts normal to the plate (which has already been taken account in the equations shown earlier).

Figure 7 shows schematically how the different forces contribute to the overall unsteady lift production in the surging wing. Figure 8 compares the theoretical model with data from the AVT-202 technical team, showing a reasonable agreement between theory and experiment. The acquisition of the experimental data is described in the complimentary session paper 'Low Reynolds Number Acceleration of Flat Plate Wings at High Incidence. ${ }^{5}$ 


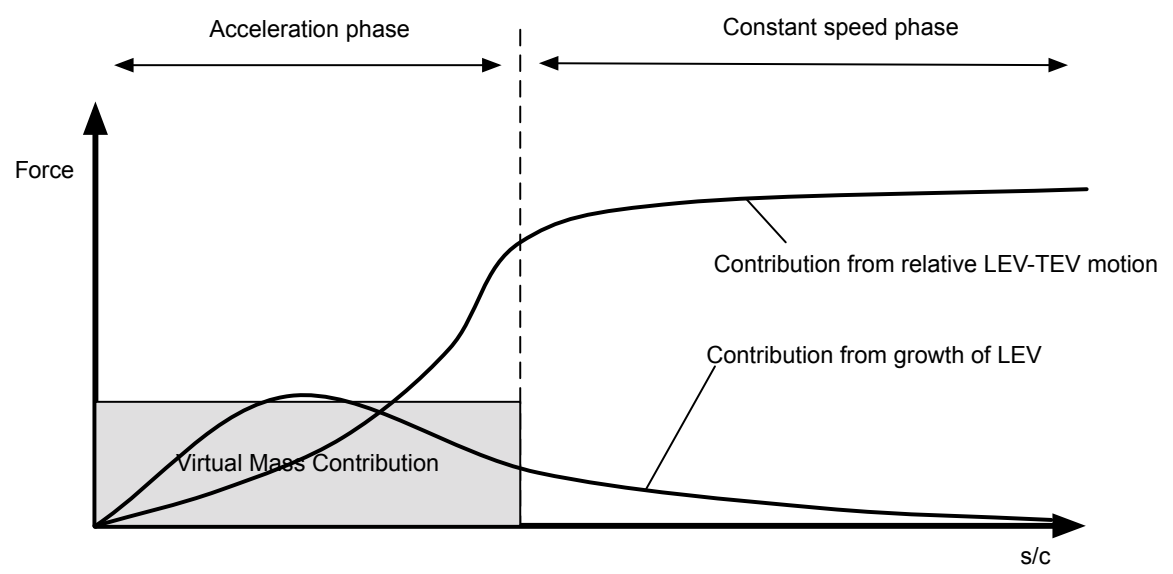

Figure 7: Schematic diagram showing the different force contributions for a surging plate.

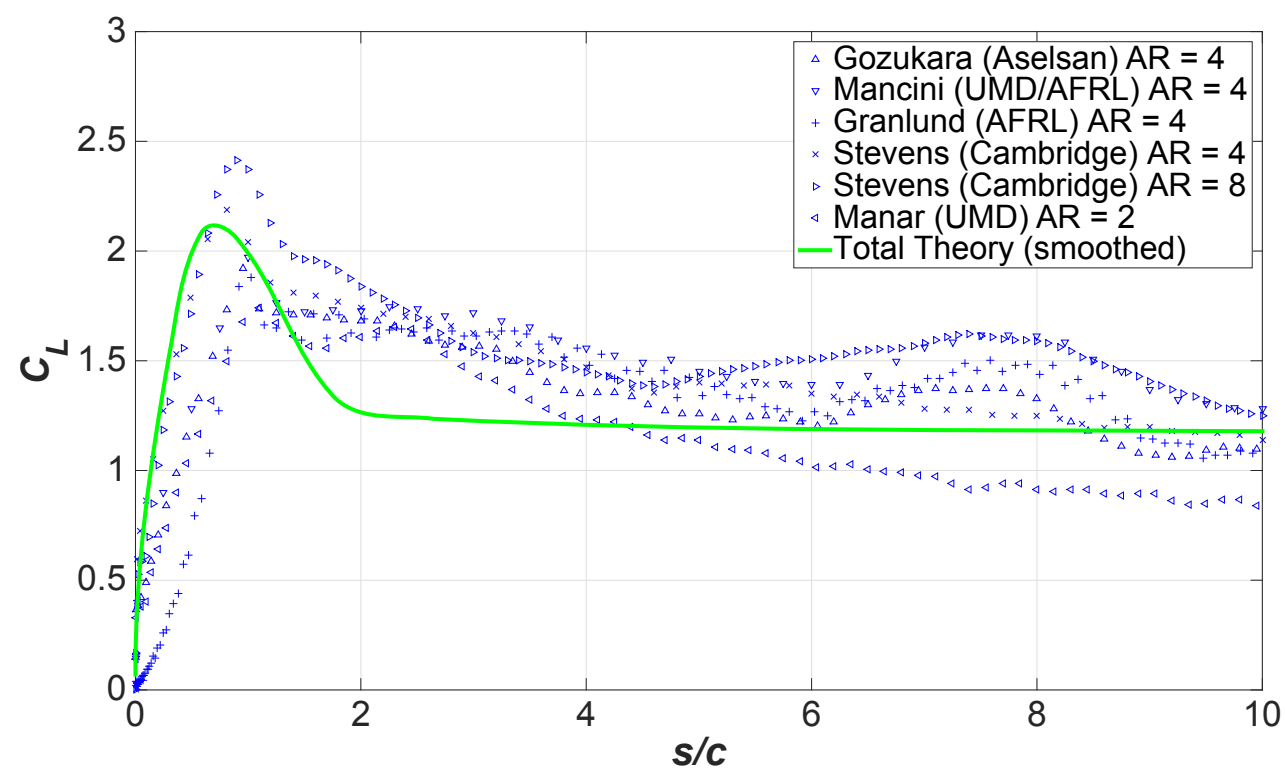

Figure 8: Comparison of AVT-202 technical team surge case data with surging theoretical model.

\section{Pitching Wing}

In pitching wings the resulting lift force can be broken down into three contributions: an inertial added mass term, a vortex lift term and an additional contribution due to pitch rate (which has the same effect as a bound vortex, as discussed below). 


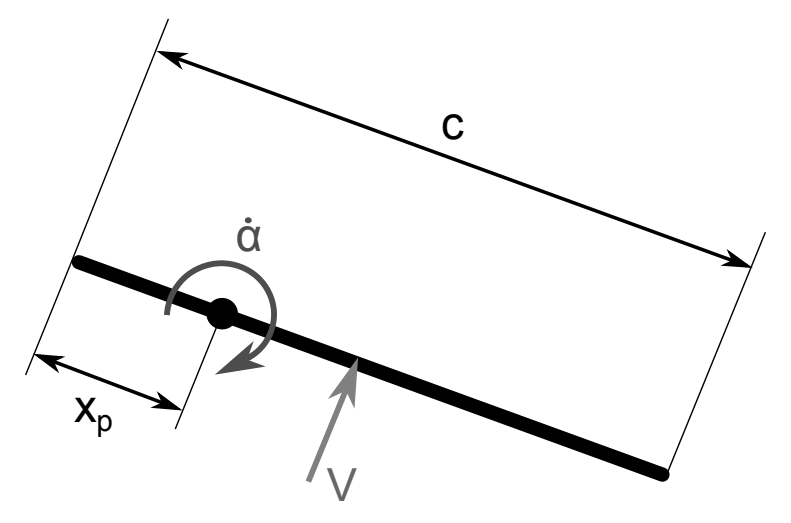

Figure 9: Coordinate system for a wing pitching about a point $P$.

Using the coordinate system shown in Figure 9 we obtain the normal velocity at mid-chord:

$$
V=\left(\frac{c}{2}-x_{p}\right) \dot{\alpha}
$$

\section{A. Non-circulatory force}

The non-circulatory forces only occur at the start and end of the pitching motion (assuming that most of the pitch is at constant rotational speed) and their magnitude depends on the 'sharpness' of acceleration at either end of the pitch. We assume the added mass potential flow to be a superposition of a rotation about the mid-chord and a plunge (normal to the plate) with the speed $V$. The former does not generate a force (but a moment about the mid-chord). The added mass contribution can be computed from the flow of a plate at $90^{\circ}$ angle of incidence and the mid-chord normal acceleration. Thus the magnitude of the non-circulatory force depends on the hinge location $x_{p}$. Rotations about the leading and trailing edges produce spikes of equal opposite magnitudes at the start and end of the motion while rotation about the centre experiences no non-circulatory force. This has been shown to be correct in AVT-202 experiments. ${ }^{6}$

Using the above expression for $V$ and the previously stated equation for the added mass force we obtain for the non-circulatory contribution to lift:

$$
C_{L, n o n-c i r c}=\frac{\pi c^{2}}{4 U^{2}}\left(1-2 \frac{x_{p}}{c}\right) \ddot{\alpha}
$$

Note that this applies at zero angle of incidence, i.e. at the start of the pitch. At the end of the pitch (when the second spike appears), the force vector is rotated by $\alpha_{\text {end }}$, thus the second spike lift magnitude is reduced:

$$
C_{L, n o n-\operatorname{circ}}=\cos \left(\alpha_{\text {end }}\right) \cdot \frac{\pi c^{2}}{4 U^{2}}\left(1-2 \frac{x_{p}}{c}\right) \ddot{\alpha}
$$

\section{B. Vortex Lift}

The growth of the LEV and TEV and their movement relative to each other generate a lift force that can be computed using the same model as discussed for the surging wing.

The strengths of the overall circulation is once again assumed to follow a modified Wagner function. Here, the modification arises from the fact that during the pitch motion the actual angle of incidence of the

wing is below the final steady- state value. Thus, the angle-of-incidence modified Wagner function can be expressed as:

$$
\frac{\Gamma(t)}{\Gamma_{\infty}}=\frac{\alpha(t)}{\alpha_{\infty}}\left[0.914-0.3151 \exp \left(\frac{s / c}{0.1824}\right)-0.5986 \exp \left(\frac{s / c}{2.0282}\right)\right]
$$




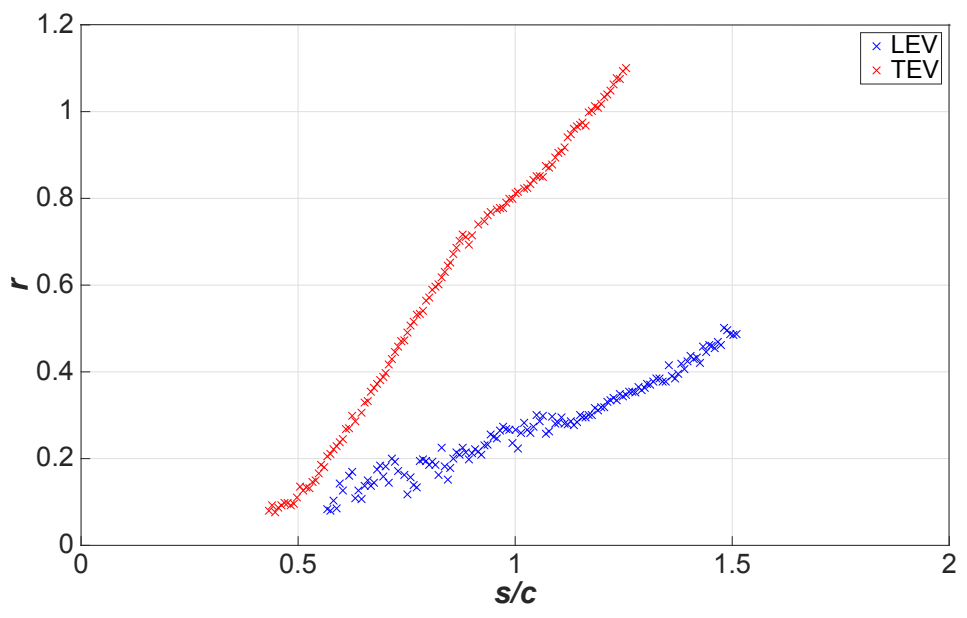

Figure 10: LEV and TEV distance from wing leading edge for fast pitch case.

which implies that the asymptotic circulation $\Gamma_{\infty}$ is proportional to the angle of incidence.

By reference to Figure 10, it is once again assumed that the relative velocity between the LEV and TEV is around half of the freestream. Similar to previous arguments, it is also suggested to use $c \cdot \cos \alpha$ as the relative LEV-TEV distance for the circulation production term in Eq. 7.

\section{Virtual quasi-steady angle of incidence}

Depending on the location of the pitch axis, the pitching motion can introduce an additional normal velocity at the mid-chord which increases the effective angle of incidence by approx. $V / U_{\infty}$. Assuming that this generates lift according to $2 \pi \alpha$ there is therefore an additional lift contribution of ${ }^{\mathrm{c}}$ :

$$
C_{L, 2}=\frac{2 \pi}{U_{\infty}}\left(\frac{c}{2}-x_{p}\right) \dot{\alpha}
$$

However, if we continue to assume that bound circulation is vanishingly small then the lift contribution due to this additional effective angle of incidence should be included in the vortex lift. We therefore propose to include this additional incidence in the correction of the Wagner function. In practice it is found that the inclusion of this additional incidence has a small effect.

\section{Additional circulatory force ('Magnus Force')}

The physical action of pitching the wing introduces a real geometric rotation into the fluid. This can be interpreted as a further bound circulation (not included in the LEV). The existence of this bound circulation (and the additional lift it generates) can be explained in two ways: Either a virtual camber caused by pitch (consider the motion of the TE relative to the LE while the fluid travels along the chord), as seen in Figure 11, or an additional circulation due to the plate rotation.

Applying unsteady thin airfoil theory to a pitching plate (see Figure 11) gives the following result for lift coefficient:

$$
c_{l}=2 \pi\left(\alpha+\frac{c \dot{\alpha}}{2 U_{\infty}}\left(\frac{3}{2}-\frac{2 x_{p}}{c}\right)\right)
$$

This includes the steady contribution of $2 \pi \alpha$ and the virtual quasi-steady angle of incidence discussed above. After removing both we are left with the virtual camber contribution:

$$
c_{l, c i r c}=\frac{\pi c}{2 U_{\infty}} \dot{\alpha}
$$

${ }^{\mathrm{c}}$ This result is also given by Theodorsen. ${ }^{7}$ 

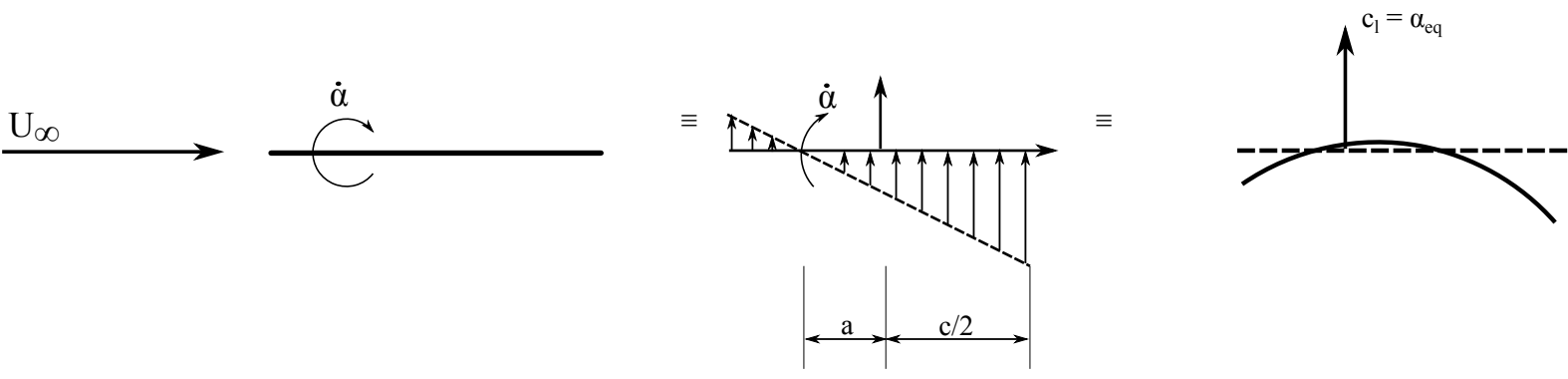

Figure 11: Virtual camber caused by a pitching wing. Adapted from Leishman. ${ }^{8}$

The same result is obtained if we assume that the pitching motion introduces an additional bound circulation:

$$
\Gamma=\frac{\pi c^{2}}{4} \dot{\alpha}
$$

which generates lift according to the Magnus effect.

The total lift is once again the sum of the various contributions discussed above. Figure 12 shows schematically how the different contributions affect the overall lift force. Figure 13 compares the pitch data from the AVT-202 technical team with the model, demonstrating that the model captures the key features of the force history.

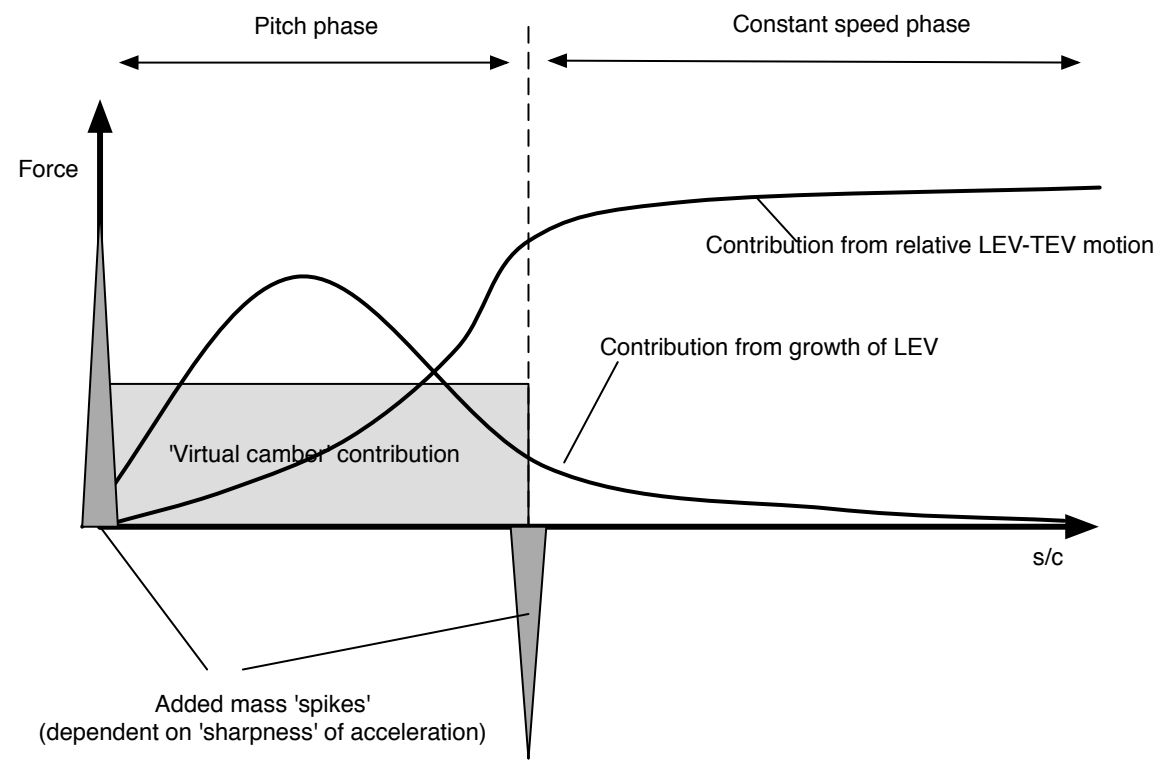

Figure 12: Schematic diagram showing the different force contributions for a pitching plate. 


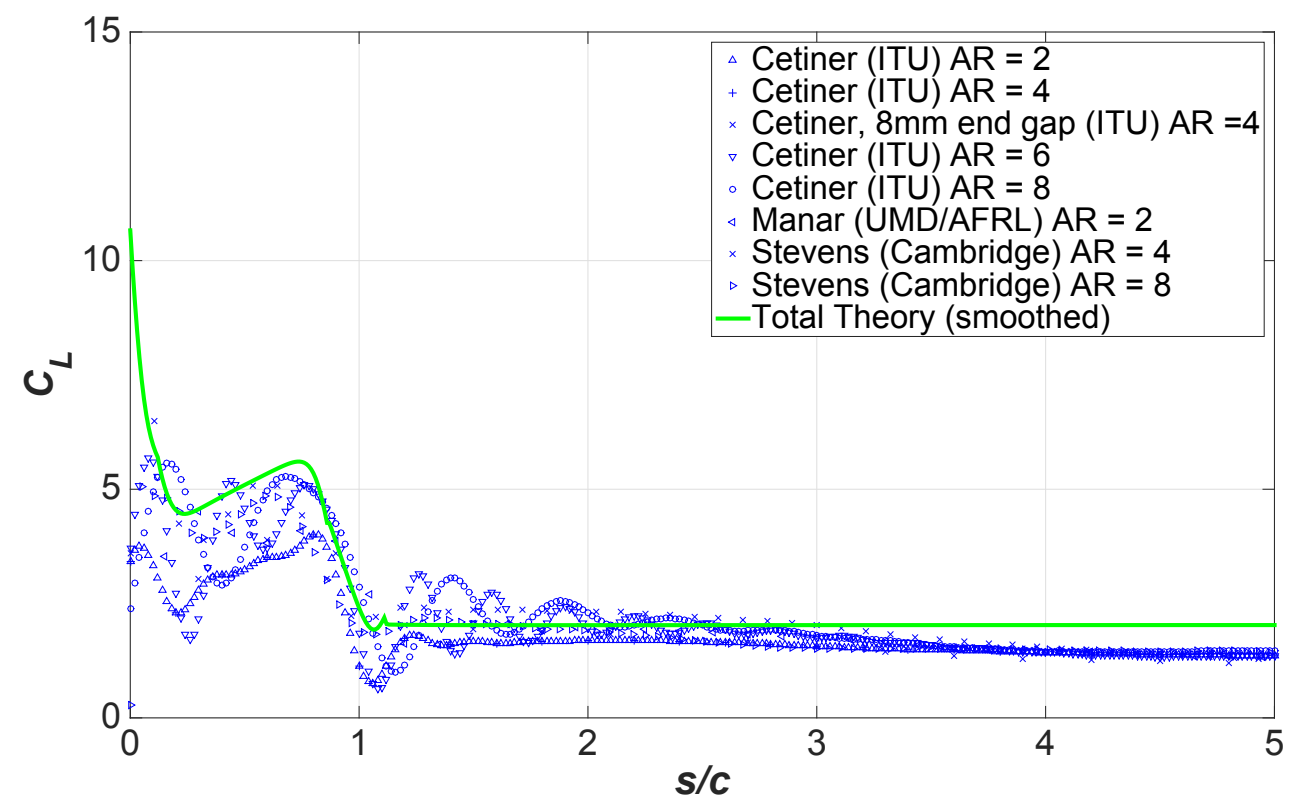

Figure 13: Comparison of AVT-202 technical team pitch case data with pitching theoretical model.

\section{Conclusions}

A simple low-order model is proposed to predict the unsteady lift force generated during the early part of an unsteadily accelerating and pitching wing. The model is valid for motions where the unsteady portion occurs over a relatively small number of chord-lengths of travel and it is expected to be capable of estimating the forces during the unsteady part of the motion as well as for a few chord lengths of travel beyond reaching steady state. The data used to inform this model has been generated as part of a NATO technical research team (AVT-202) where surging and pitching flat plates with a steady state angle of incidence of $45^{\circ}$ where investigated in incompressible flow at a range of Reynolds numbers.

The main aim of this model is not to accurately predict the lift force but to identify a number of viscous and inviscid force production mechanisms so as to help the understanding of unsteady low Reynolds number flows. In particular, the model identifies various circulatory and non-circulatory forces which illuminates the connection between unsteady force production and wing kinematics.

For very fast motions it is seen that the instantaneous force during the acceleration phase is dominated by a virtual mass term (or added mass) which can be predicted relatively easily from wing kinematics. For less fast motions (and at other times in the cycle) there are a number of circulatory forces whose contribution depends on the strengths and relative locations of various vortices in the flow. By combining all vortices into a single representative leading edge vortex (LEV) and its trailing edge counterpart (TEV) a reasonably good estimate of the effective lift force can be obtained. For pitching wings there is an additional force contribution due to the plate rotation which can be interpreted either as a Magnus force or a virtual camber.

\section{References}

${ }^{1}$ Noca, F., “Personal Correspondance," 2015.

${ }^{2}$ Lamb, H., Hydrodynamics, Cambridge University Press, Cambridge, United Kingdom, 6th ed., 1932.

${ }^{3}$ PittFord, C. W. and Babinsky, H., "Lift and the leading-edge vortex," J. Fluid Mech, Vol. 720, 2013, pp. 280-313.

${ }^{4}$ Wagner, H., "Über die Entstehung des Dynamischen Auftriebes von Tragflügeln," Zeitschrift für Angewandte Mathematik und Mechanik, Vol. 5, 1925, pp. 17-35.

${ }^{5}$ Stevens, P. R. R. J., Babinsky, H., Manar, F. H., Mancini, P., Jones, A., Granlund, K., Ol, M. V., Bomphrey, R., and Gozukara, A. C., "Low Reynolds Number Acceleration of Flat Plate Wings at High Incidence," AIAA SciTech 2016, 2016.

${ }^{6}$ Cetiner, O., Stevens, P. R. R. J., Jones, A. R., Bernal, L., and Gozukara, A. C., "Parametric variations in aspect ratio, leading edge and planform shapes for the rectilinear cases of AVT-202," AIAA SciTech 2016, 2016.

${ }^{7}$ Theodorsen, T., “General Theory of Aerodynamic Instability and the Mechanism of Flutter,” Tech. Rep. 496, NACA, 1934.

${ }^{8}$ Leishman, J. G., Principles of Helicopter Aerodynamics, Cambridge Aerospace Series, Cambridge University Press, 2000. 